\title{
MASTER/SLAVE: Interprocessor communications between PDP-8s
}

\author{
BRUCE E. HAMILTON and TIMOTHY F. ELSMORE \\ Walter Reed Army Institute of Research, Washington, D.C. 20012
}

\begin{abstract}
This paper describes an inexpensive, powerful, and easily learned interprocessor communication system for PDP-8 computers. When executed, the master machine exercises control over the slave using commands similar to DEC's OS/8 copy program, FOTP.
\end{abstract}

In the late 1960s, if a psychologist was fortunate enough to have a minicomputer system, it was expensive and likely to have limited capabilities. Presently, technological breakthroughs have brought the price of computer systems down to the point where a relatively large number of psychologists have not only on-line computer systems, but multiple systems with powerful peripheral devices. This pleasant development has brought not only advances in on-line control, but also some problems. One of these, communication of information between computers, is the focus of this paper.

In the early years of minicomputer use, the task of interprocessor communications was relatively simple, but painful. Most systems were configured with limited memory and paper tape. Almost without exception, early minicomputers used one of a few standard codes for paper tape, and the only problem in communicating between CPUs was whether or not the codes were compatible. Interprocessor communication by paper tape was a matter of stamina more than hardware or software. As the minicomputer industry grew, highspeed mass storage devices replaced paper tape in interprocessor communications. Due to price and improving technology, systems developed where each used the latest peripheral storage devices available at the time of purchase. Media compatibility and data format were frequently sacrificed. While always theoretically possible, the alternative of communicating directly between CPUs via hard lines was rarely attempted.

\section{INTERPROCESSOR COMMUNICATION OPTIONS FOR THE PDP-8}

The DEC PDP-8 has frequently been employed in laboratory applications, and DEC has produced several hardware modules capable of supporting interprocessor communications. The most complicated of these was the Programmed Data Mover, a sophisticated direct memory access device that is no longer available. The least complicated of these modules is the DR8E 12-bit I/O module. DEC's best attempt is probably the DB8E interprocessor buffer module. Others (see below) have also used KL8J asynchronous data control cards as $\mathrm{I} / \mathrm{O}$ ports for interprocessor communications. Unfortunately, although several modules (including modems) allow access to the CPU and have the potential needed, the lack of versatile software has precluded practical use. Some software is available from DEC, but most programs are user written. DEC's main software thrust has been to include the PDP-8 in its DECNET software system. Unfortunately, although DECNET uses a variety of hardware modules, it is designed to be run as a task of RTS-8, DEC's timesharing system. Besides having to learn the DECNET protocols (set up mostly for industrial use), one must learn how to utilize RTS-8, a tedious and often difficult programming task. The DECNET software also requires $20 \mathrm{~K}$ of memory, which, in the PDP-8, may be required for other tasks.

A communications package called IPSLAV by Karlheinz Siebold is available from DECUS (8-832). This program provides the user with a device handler for the DB8E module and a slave program for the receiving machine. Unfortunately, the program lacks the flexibility that is necessary to make the task of interprocessor communications practical.

Robert Phelps of the University of Rochester has developed a program called DEC10 (submitted to DECUS) that allows him to communicate on-line between a PDP-8 and a PDP-10 via KL8J modules and modems. With minor modifications, this program can send information via a $K L 8 J$ to a second $K L 8 J$ in a machine that is programmed to use that $\mathrm{KL} 8 \mathrm{~J}$ as the terminal device. Thus the information sent via $\mathrm{KL} 8 \mathrm{~J}$ modules is received by the second machine as though it were being typed by a user at the keyboard. All transfers via the TTY of the second machine are read by the first. Unfortunately, this requires the slave machine to be in the RTS-8 environment, something that is an undesirable restriction.

Given the drawbacks of the above options, we decided to write a pair of programs that would: (1) not require someone to be present at both CPUs, (2) be capable of more than just transferring files, (3) make use of the FOTP features of wild cards, (4) run under timeshared RTS-8 or stand-alone, (5) require little or no software overhead, and (6) run under BATCH control. We also decided that it should utilize either the DR8E or DB8E modules, two low-cost devices. The end result was a pair of programs we call MASTER/SLAVE. 


\section{MASTER/SLAVE}

MASTER/SLAVE is actually two PAL8 source files that are conditionally assembled for either the DB8E or DR8E modules by setting either the DB8E or DR8E parameter to 1 , dependent upon the device selected. The device code is set by setting parameter DVCODE equal to the device code desired. The source is then assembled via PAL8 and loaded, creating coreimage files of both MASTER and SLAVE that run in $8 \mathrm{~K}$ core. These programs will run in any OS/8 environment, including as a background OS/8 program in RTS-8.

Which CPU is to be the master and which the slave is determined merely by which program is selected for either CPU. That is, either CPU may be the master so long as the other is the slave. When called, each program waits for the other CPU to be loaded and then performs a handshake verification of the interprocessor hardware link. When the link is complete, the keyboards prompt the user to indicate that the link is established. At this point, the slave CPU awaits instructions from the master CPU and does not require operator assistance. As an aid, all actions taken by the master CPU that concern the slave are typed out as a $\log$ on the slave's terminal. The slave's keyboard is also responsive to control $\mathrm{C}(\uparrow \mathrm{C})$ and control $\mathrm{P}(\uparrow \mathrm{P})$ in the same manner as the master (see below).

After the handshake, the master CPU responds with an asterisk. This asterisk is a simulation of the standard OS/8 command decoder, with some modifications. The Appendix gives examples of the operation of MASTER/SLAVE. The standard OUTPUT <INPUT format of the command decoder is retained, although up to 10 input files may be specified, with the output file names being the same as the input names by default. Thus the following format is legal, *SYS: $<$ FILE1, FILE2, SYS:FILE3, indicating that three files on the master (two from DSK: and one from SYS:) are to be transferred to the SYS: device of the slave under the same name as given in the input specification. Effectively, all output naming is by default from the input specification. Transfer can also be made, with the output file receiving a different name by explicitly specifying a file name. Unfortunately, only one output device or file name is allowable on each line.

MASTER/SLAVE responds to either $\uparrow C$ or $\uparrow P$. The $\uparrow C$ works exactly the same as in OS/8, namely, causing an exit to the OS/8 monitor on both CPUs. The $\uparrow P$, however, causes the master to exit to $\mathrm{OS} / 8$, with the slave directed to await a new handshake. Thus, the user is able to type $\uparrow P$ on the master (or slave), exit to $\mathrm{OS} / 8$, perform any task available to him, and then reestablish the link merely by calling the same program again. Additionally, rubouts, linefeeds, and control $\mathrm{U}(\uparrow \mathrm{U})$ work as in DEC's command decoder.

As transfer is assumed to be between CPUs, two options control the direction of transfer, the /I and the
/O options. Specifying the /I option means that the input specification refers to the slave and output is to the master. Conversely, the $/ 0$ option (the default) means that output is to the slave and input is from the master. As a convenience, the back arrow $(<)$ need not be specified when output is to the DSK: of the slave. Thus, the command line *FILE1,FILE2 specifies that FILE1 and FILE2 on the DSK: of the master are to be transferred to the DSK: of the slave; this is equivalent to the command *DSK: $<$ FILE1,FILE2/O.

Wild cards also have been implemented. The conventions of "*" and "?" are used exactly as in DEC's FOTP program. For example, specifying *SYS:<*.PA causes all files with the extension .PA to be transferred to the SYS: device of the slave. As an aid, the /Q option is implemented as in FOTP and allows the user to select files from the possible ones selected via wild cards. Additionally, each of the 10 files allowed as input (see above) may contain wild card specifications, although the /Q option, when specified, would apply to each instance of wild card.

The ability to access the directory of any file-oriented device is also available. By specifying the /S option, the directory of the slave's DSK: can be listed in a format identical to that found with the CCL command .DIR DSK:/E $=1$. Devices other than DSK: can be directoried by specifying the device followed by /S. In the same manner, the master's devices can be directoried by using the $/ \mathrm{M}$ option. In addition, files on the slave may be deleted by specifying the device and file name, followed by /D. Typing control O ( $\uparrow)$ any time during the printing of a directory will terminate the printing.

Two other options are available. The first is /E, which may be used with the $/ O$ option to output and execute the specified SAVE format (.SV) file on the slave machine. If the file is already on the slave's system device, the /E option alone will execute the program. The second option is the /B option. This option, used either alone or in conjunction with the /O option, causes BATCH to be loaded and the specified file to be submitted as the BATCH job. For instance, the command *FILE1.BI/B/O transfers file FILE1.BI to the slave and submits it to BATCH, while *FILE1.BI/B assumes that FILE1.BI is already on the slave. Both commands assume that BATCH.SV is already on the slave's system device. MASTER/SLAVE is completely compatible with BATCH. Any operation can be conducted under BATCH, with the exception of responses to the $/ Q$ option (used with wild cards). Furthermore, the BATCH convention of using the dollar sign (\$) as a substitute for the ESC key is retained here as a substitute for $\uparrow \mathrm{C}$ after execution of the current command line, and the pound sign (\#) is used to emulate the $\uparrow P$ option after execution of the current command line.

MASTER/SLAVE has proven to be easily learned due to the close adherence to DEC conventions. Errors that 
might occur (such as specifying a nonexistent file) are reported on both the master and slave terminals in English and are self-explanatory. If the computer completes the transfer without an error message, then complete confidence can be placed in the received information, as each 12-bit word transferred is verified by echoing it back to the sending machine for comparison. After each block of information has been transferred, each machine reports to the other whether or not the transfer was error free. If an error were to occur, the block would be retransmitted up to three times before a transmission error would be reported.

In addition to numerous one-time file transfers, MASTER/SLAVE is used routinely to archive data stored on RX01 floppy disks in one laboratory on a TU10 magnetic tape in another laboratory. Although in a timesharing environment it may take up to $4 \mathrm{sec} /$ block to transfer, due to the ability to use wild cards and BATCH, transfers can be set in motion and left to finish by themselves. This represents a tremendous time-saver, as laboratory personnel can easily attend to other tasks without worry. MASTER/SLAVE is also capable of remote processing of data. If both machines were in BATCH, one with a call to MASTER and the other to SLAVE, then one machine could wait for the other, transfer data, leave MASTER/SLAVE, process data, and return with information or for further jobs.

Machines in different laboratories, with different configurations of peripherals and different schedules of use, can be interconnected to optimize their capabilities. For instance, data collected on one machine can be plotted on another machine with an X-Y plotter, or if one machine is idle at night, it can be put to use for off-line data analysis. Generally, the maximum cable distance for DEC devices is $100 \mathrm{ft}$; however, with some minor electronics, the distance can be increased to $250 \mathrm{ft}$.

The flexibility, ease, and power of MASTER/SLAVE make it a useful and time-saving aid to the management of computerized laboratories. The system not only makes use of the device independence of OS/8 programming, but carries it one step further to machine independence.

\section{Appendix}

*FILE1<FILE1/O This command transfers the file FILE1 from the master to the slave.

*FILE1 $<$ FILE1/I This command transfers the file FILE1 from the slave to the master.

*FILE1,FILE2 This command transfers the files FILE1 and FILE2 from the master to the slave.

*FILE2<FILE1 This command transfers the file FILE1 from the master to the slave under the name FILE2.

*RXA1:<*.PA This command transfers all files on the master with the extension .PA to the slave's floppy disk drive 1 .

*DATA.BI/B/O This command transfers the BATCH file DATA.BI from the master to the slave and submits it to BATCH.

*SYS:/S This command directories the SYS: device of the slave.

**.DA, *.LD/Q This command transfers all files on the master with the extensions .DA and .LD if the letter " $Y$ " is typed in response to the prompt.

*LOSER.BD/D This command deletes the file LOSER.BD on the slave.

*DELAY.SV/E This command loads the file DELAY.SV found on the slave's SYS: device and chains to it for execution.

*UNK???.PA This command transfers all files beginning with the letters UNK, regardless of the final three letters, and with the extension of .PA. 